\title{
Outcome of Robot-Assisted Radical Cholecystectomy in a High-Volume Tertiary Cancer Center in India
}

\author{
Mahesh Goel, MS \\ GI and HPB Service, Department of Surgical Oncology, Tata Memorial Hospital, Mumbai, India. \\ E-mail: drmaheshgoel@gmail.com
}

Sagar R. Kurunkar, MS, DNB

GI and HPB Service, Department of Surgical Oncology, Tata Memorial Hospital, Mumbai, India.

Amol Kanetkar, MS, Mch

GI and HPB Service, Department of Surgical Oncology, Tata Memorial Hospital, Mumbai, India.

\section{Shraddha Patkar, MS, Mch}

GI and HPB Service, Department of Surgical Oncology, Tata Memorial Hospital, Mumbai, India.

Mary Ann Liebert, Inc. DOI: 10.1089/vor.2018.0539
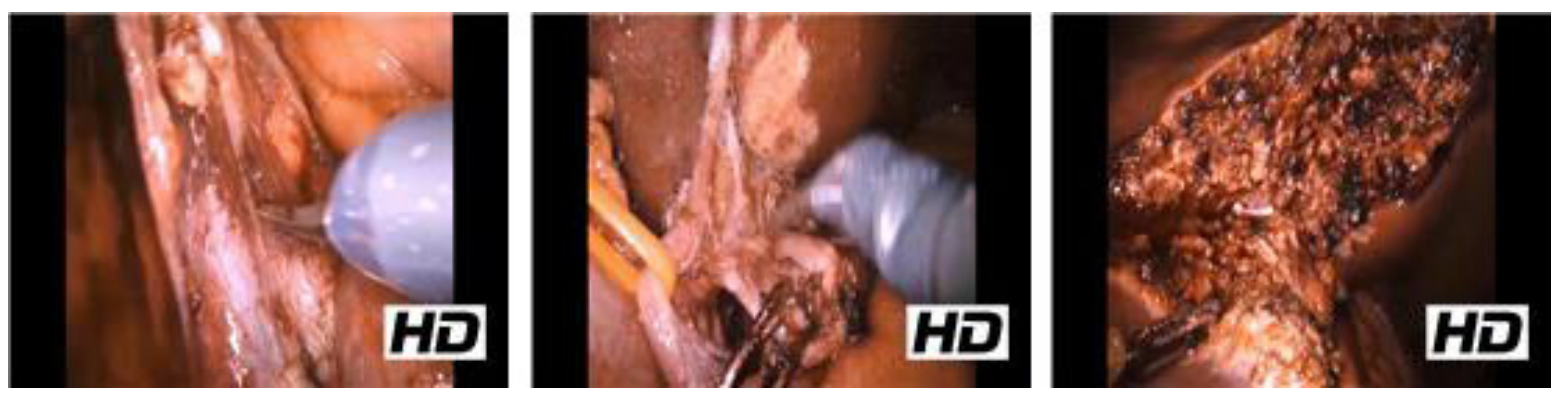

\section{Abstract}

Introduction: Minimally invasive radical cholecystectomy is a complex laparoscopy. Robotic surgery is now an option to complete a radical cholecystectomy because of its high definition, magnified threedimensional view of the operative field, and articulating instrumentation. ${ }^{1-3}$ Robotic surgery enables a safe dissection in otherwise difficult to access areas such as the porta hepatis. This video reviews the role of robotic surgery in the management of gall bladder (GB) malignancy.

Methods: A 28-year-old lady, with no comorbidities, presented with abdominal pain and underwent an evaluation with a contrast-enhanced CT scan of chest and abdomen. The CT scan revealed a mass in the GB with no evidence of distant metastases. Liver function tests were normal and a CA19-9 was $898 \mathrm{U} / \mathrm{mL}$. A robotic radical cholecystectomy using five ports (four robotic and one assistant port) was performed. The procedure started by clearing the hepatoduodenal ligament nodes (stations 8,12 , and 13 with interaortocaval node sampling). The triangle of Calot was then dissected and secured with clips. Next a wide excision of segments $4 \mathrm{~b}$ and 5 was performed including the GB. The complete specimen was extracted in a bag from a small incision at the assistant port.

Results: The procedure was performed in 330 minutes with a blood loss of $200 \mathrm{~mL}$. There were no perioperative complications and the postoperative stay was 3 days. Final histopathology report revealed moderately differentiated adenocarcinoma of GB invading serosa (pT3) with negative margins and 4 out of 14 lymph nodes showed presence of metastases. The overall cohort shows 22 robotic radical cholecystectomies for GB malignancy. The median age was 53 years. The average duration of surgery was 270 minutes with a median blood loss of $120 \mathrm{~mL}$. The median postoperative stay was 4 days and the median nodal yield for radical cholecystectomy was 8 . The overall median survival at 18 months was $100 \%$ with one recurrent hepatic lesion. 
Discussion: Robotic radical cholecystectomy may offer technical superiority over laparoscopic surgery and is an oncologically acceptable approach with good short-term oncologic outcomes. This type of surgery may require a highly specialized center with adequate experience in hepatopancreatobiliary surgery.

No competing financial interests exist.

Runtime of video: 9 mins 5 secs

Keywords: robotic radical cholecystectomy, gallbladder cancer, outcomes

(c) Mahesh Goel et al., 2019; Published by Mary Ann Liebert, Inc. This Open Access article is distributed under the terms of the Creative Commons License (http://creativecommons.org/licenses/by/4.0), which permits unrestricted use, distribution, and reproduction in any medium, provided the original work is properly cited.

\section{Cite this video}

Mahesh Goel, Sagar R. Kurunkar, Amol Kanetkar, Shraddha Patkar, Outcome of Robot-Assisted Radical Cholecystectomy in a High-Volume Tertiary Cancer Center in India, Videoscopy. 2019, DOI: $10.1089 /$ vor.2018.0539.

\section{References}

1. Shen BY, Zhan Q, Deng XX, et al. Radical resection of gall bladder cancer: Could it be robotic? Surg Endosc 2012;26:3245-3250.

2. Georgakis GV, Novak S, Bartlett DL, Zureikat AH, Zeh HJ, Hogg ME. The emerging role of minimally invasive surgery for gallbladder cancer: A comparison to open surgery. SM Min Inv Surg 2017;1:1007.

3. Chandarana M, Patkar S, Tamhankar A, Garg S, Bhandare M, Goel M. Robotic resections in hepatobiliary oncology-Initial experience with Xi da Vinci system in India. Indian J Cancer 2017;54:52-55.

Original Publication Date: 2019 